\title{
Optical coherence tomography evidence of macular ganglion cell- inner plexiform layer thinning in eyes with subretinal drusenoid deposits
}

\author{
Solmaz Abdolrahimzadeh ${ }^{1} \cdot$ Francesco Parisi ${ }^{2} \cdot$ Michela Marcelli $^{2} \cdot$ Rosalia Giustolisi $^{2} \cdot$ Magda Gharbiya $^{2}$
}

Received: 7 October 2018 / Revised: 30 January 2019 / Accepted: 1 March 2019 / Published online: 29 March 2019

(c) The Royal College of Ophthalmologists 2019

\begin{abstract}
Background/Objectives The purpose of this study was to evaluate macular ganglion cell layer-inner plexiform layer (GCL-IPL) and choroidal thickness in early age-related macular degeneration (AMD) in eyes with subretinal drusenoid deposits (SDD).

Subjects/Methods Comprehensive ophthalmological examination was performed. Near infrared reflectance and raster images using enhanced depth imaging were acquired with spectral domain optical coherence tomography. Drusen and SDD were diagnosed based on raster scans and near infrared reflectance. GCL-IPL maps were generated with automated segmentation and choroidal thickness maps were obtained by manually delineating the choroid-scleral boundary.

Results Forty-eight eyes from 48 patients (mean age 77.5 \pm 5.7 , range 68-90 years) with a diagnosis of early AMD and 42 eyes of 42 age-matched control subjects (mean age 76.9 \pm 5.7 , range 67-88 years) were included. Of these, 28 eyes $(58.3 \%)$ had drusen alone, 4 eyes (8.3\%) had SDD alone, and 16 eyes (33.3\%) had drusen associated with SDD. Compared with controls, average choroidal thickness was significantly decreased in AMD eyes $(P<0.05)$. There was no significant difference in choroidal thickness in eyes with SDD with respect to those with drusen alone. GCL-IPL thickness was reduced in an annular pattern at the 3 and $6 \mathrm{~mm}$ macular areas in AMD patients with respect to controls $(P<0.05)$. GCL-IPL thickness at $3 \mathrm{~mm}$ was significantly reduced in eyes with SDD with respect to those with drusen alone $(P=0.03)$.

Conclusions The GCL-IPL is reduced in thickness with an annular pattern in early AMD and is significantly thinner in eyes with SDD.
\end{abstract}

\section{Introduction}

Age-related macular degeneration (AMD) is one of the foremost causes of legal blindness in developed countries. Genetic and environmental risk factors together with oxidative stress are among the multifactorial pathogenic factors involved in this condition [1]. AMD predominantly involves the outer retinal layers, where extracellular accumulation of lipoprotein in drusen is associated with photoreceptor loss

Solmaz Abdolrahimzadeh

solmaz.abdolrahimzadeh@uniroma1.it

1 Ophthalmology Unit, NESMOS Department, St. Andrea Hospital, "Sapienza" University of Rome, Rome, Italy

2 Ophthalmology Unit, Department of Sense Organs, Azienda Policlinico Umberto I, "Sapienza "University of Rome, Rome, Italy and thinning of the outer nuclear layer. However, inner retinal layers, including the vascularized layers, such as the retinal nerve fibre, ganglion cell, inner plexiform, inner nuclear, and outer plexiform layers may also be involved following photoreceptor degeneration [2, 3]. Recent classification of early AMD differentiates lipoprotein extracellular accumulations in drusen localized below the retinal pigment epithelium from subretinal drusenoid deposits (SDD) localized above the retinal pigment epithelium [4-6]. Drusen are typically found in areas where cone density is higher in the central macula contrary to the preferential location of SDD in rod rich areas; superior and inferior to the macula $[5,7,8]$. However, SDD are frequently observed in conjunction with drusen in the context of AMD [8-10].

The pathogenesis of SDD, otherwise called reticular pseudodrusen, is not completely understood, however, numerous authors have found a reduction of choroidal thickness $[5,6,11,12]$. The ganglion cell-inner plexiform layer (GCL)-(IPL) has been reported to be thinner in AMD 
[2], but this layer has not been studied in eyes with SDD. The vascularized retinal layers correlate positively with choroidal thickness in normal eyes [13], owing to this correlation, the present study aimed to evaluate both the choroidal and macular GCL-IPL thicknesses in the presence of SDD in an attempt to further understand the pathogenetic mechanisms involved.

\section{Subjects and methods}

This was a prospective study where patients with early AMD and age-matched healthy control subjects were enrolled. The research was approved by the Ethical Board of the "Sapienza" University of Rome and was carried out according to the tenets of the Declaration of Helsinki. All patients and subjects were informed of the study and gave their written consent.

Inclusion criteria were patients with drusen and SDD assessed by near infrared reflectance and spectral domain optical coherence tomography (SDOCT) scans by two investigators. Diagnosis of SDD was based on previous guidelines on evidence of at least five SDD in more than one raster line scan [14-16]. Diagnosis of drusen was made when soft drusen were found in the central macular area. Exclusion criteria were eyes with choroidal neovascular membranes or geographic atrophy. Both patients and control subjects were excluded from the study in the presence of glaucoma, systemic pathology, ocular disease, or pharmacological therapy, which could affect retinal or choroidal thickness values, history of previous ocular surgery, spherical equivalent beyond 3 diopters, or optical media opacity that could interfere with SDOCT examination.

Best-corrected visual acuity measurements were performed with early treatment diabetic retinopathy study (ETDRS) charts. Comprehensive ophthalmological evaluation of all patients and healthy controls included slit lamp examination, intraocular pressure measurement, and dilated fundus ophthalmoscopy. Axial length was evaluated with biometry (IOL Master 500 (Carl Zeiss Meditec, Dublin CA). Spectralis OCT (Family Acquisition Module, V 6.0.11.0 Heidelberg Engineering, Germany) was used to obtain near infrared images and macular scans with the enhanced depth imaging mode with the raster $20^{\circ} \times 20^{\circ}, 25$ line scan protocol with an interval between scans of $250 \mu \mathrm{m}$ and 100 frames averaged for each scan. In order to avoid bias due to diurnal changes in choroidal thickness, examinations were performed between 12:00 noon and 3:00 pm and measurements were carried out in a semi automatic method by delineating the choroidal boundaries in a method described in previous publications $[13,17]$. In this manner it was possible to obtain thickness maps using the automated software. We did not calculate the interclass correlation coefficient for measurements as our previous publications in normal and pathological eyes in detecting the choroidscleral junction showed values of above 0.95 [18].

The ETDRS macular grid at 1,3 , and $6 \mathrm{~mm}$ was centred on the fovea to evaluate retinal and choroidal thickness maps in all sectors. The automated segmentation protocol of the Spectralis OCT (Heidelberg Eye Explorer V1.9.10.0) was used to measure retinal layer thicknesses according to the IN. OCT Consensus guidelines for landmarks in OCT [19] and segmentation was evaluated by two experienced investigators to correct for any misalignment. Thickness measurements for all retinal layers and the choroid in the 9 sectors of the ETDRS macular grid at 1,3 , and $6 \mathrm{~mm}$ were recorded.

\section{Data analysis}

Statistical analysis was performed with the SPSS for windows (V 17.0, SPSS). One eye of each patient with AMD or each healthy control subject was used to perform the analysis. Normal distribution of data was analyzed by the Kolmogorov-Smirnov test. Parametric variables were compared using the unpaired $t$-test or the analysis of variance (ANOVA), as appropriate. Levene's test was used to verify variance homogeneity. Non-parametric distributed values were analyzed by the Mann-Whitney rank sum test. Categorical variables were compared using Fisher's exact test. OCT measurements between groups were compared using the general linear model, including age, sex, axial length, and spherical equivalent as covariates. $P$-values of less than 0.05 were considered as statistically significant.

\section{Results}

One eye of 48 patients (mean age $77.5 \pm 5.7$, range $68-90$ years) with a diagnosis of early AMD and one eye of 42 age-matched control subjects (mean age $76.9 \pm 5.7$, range 67-88 years) were included in this study. Figure 1 shows a near infrared image with a superimposed raster scan and cross-sectional SDOCT images of SDD lesions. We found that 28 eyes $(58.3 \%)$ had drusen alone, 4 eyes $(8.3 \%)$ had SDD alone, and 16 eyes (33.3\%) had drusen associated with SDD. Demographic characteristics of patients and controls are shown in Table 1.

Compared with controls, average choroidal thickness was significantly decreased in early AMD eyes at every location (Table 2) with a percentage reduction of $24.3 \%$ at $1 \mathrm{~mm}, 24.1 \%$ at $3 \mathrm{~mm}$, and $17.8 \%$ at $6 \mathrm{~mm}(P<0.05$, adjusted for age, sex, axial length, and spherical equivalent). However, there was no significant difference in choroidal thickness between eyes with drusen alone and those associated with SDD $(P>0.05$, adjusted for age, sex, axial length, and spherical equivalent). 
Fig. 1 Near infrared reflectance image with raster scan and cross-sectional image of subretinal drusenoid deposits using spectral domain optical coherence tomography. Deposition of granular hyperreflective material above the retinal pigment epithelium on cross-sectional image is shown. Deposits intrude on the ellipsoid zone and external limiting membrane bands

Table 1 Demographic and clinical data of patients and healthy control subjects
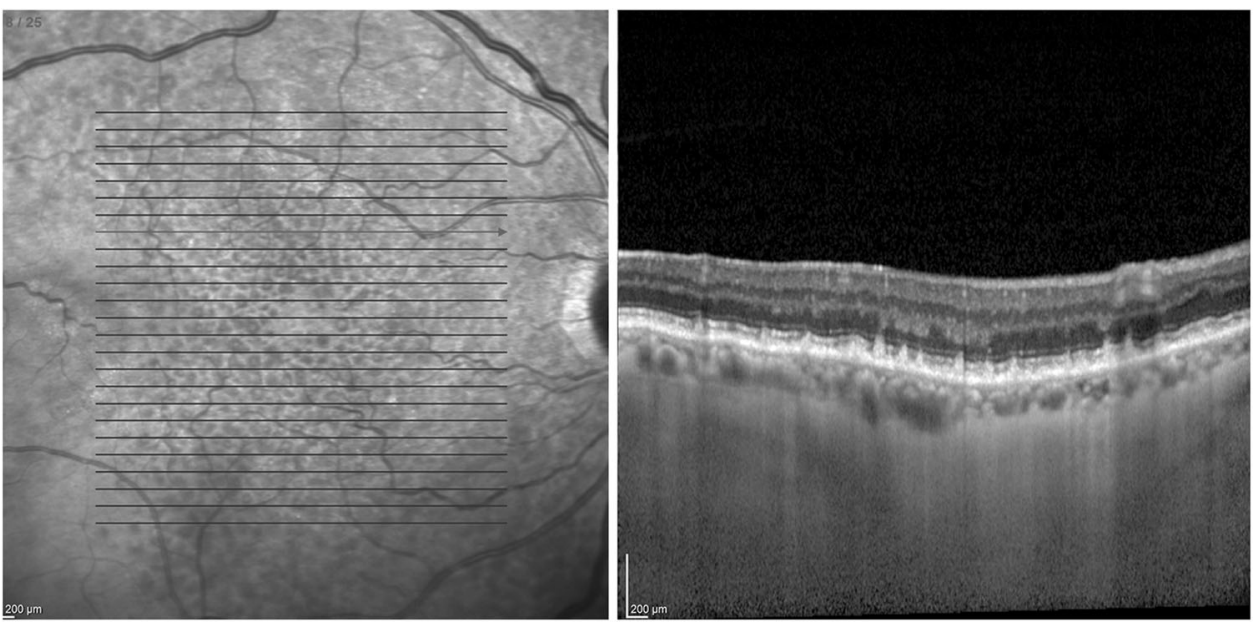

\begin{tabular}{|c|c|c|c|c|c|c|}
\hline & \multicolumn{3}{|c|}{ Early AMD eyes $(n=48)$} & \multirow[t]{2}{*}{$P$-value ${ }^{\mathrm{a}}$} & \multirow{2}{*}{$\begin{array}{l}\text { Control eyes } \\
(n=42)\end{array}$} & \multirow[t]{2}{*}{$P$-value ${ }^{\mathrm{b}}$} \\
\hline & All & $\begin{array}{l}\mathrm{CD} \\
(n=28)\end{array}$ & $\begin{array}{l}\text { SDD } \\
(n=20)\end{array}$ & & & \\
\hline Age (years) & $77.5 \pm 5.7$ & $77.1 \pm 6.6$ & $78.0 \pm 4.3$ & $0.6^{\mathrm{c}}$ & $76.9 \pm 5.7$ & $0.6^{\mathrm{c}}$ \\
\hline Gender $(\mathrm{m} / \mathrm{f})$ & $23 / 25$ & $16 / 12$ & $7 / 13$ & $0.2^{\mathrm{d}}$ & $16 / 26$ & $0.4^{\mathrm{d}}$ \\
\hline $\begin{array}{l}\text { Spherical equivalent } \\
\text { (dioptres) }\end{array}$ & $+0.5 \pm 1.3$ & $+0.6 \pm 1.6$ & $+0.5 \pm 0.9$ & $0.7^{\mathrm{c}}$ & $+0.8 \pm 1.7$ & $0.4^{\mathrm{c}}$ \\
\hline Phakic/Pseudophakic & $24 / 24$ & $13 / 15$ & $11 / 9$ & $0.8^{\mathrm{d}}$ & $23 / 19$ & $0.7^{\mathrm{d}}$ \\
\hline Axial length (mm) & $23.5 \pm 1.1$ & $23.5 \pm 0.9$ & $23.5 \pm 1.4$ & $0.8^{\mathrm{c}}$ & $23.7 \pm 0.5$ & $0.3^{\mathrm{c}}$ \\
\hline BCVA (logMAR) & $\begin{array}{l}+0.08 \pm \\
0.1\end{array}$ & $\begin{array}{l}+0.06 \pm \\
0.1\end{array}$ & $+0.1 \pm 0.2$ & $0.4^{\mathrm{c}}$ & $+0.006 \pm 0.07$ & $0.0001^{\mathrm{c}}$ \\
\hline
\end{tabular}

$B C V A$ best-corrected visual acuity

${ }^{a}$ Data analysis results comparing eyes with conventional drusen (CD) vs. eyes with subretinal drusenoid deposits (SDD)

${ }^{\mathrm{b}}$ Data analysis results comparing eyes with age-related macular degeneration (AMD) vs. control eyes ${ }^{c}$ Unpaired $t$-test with Levene's correction

${ }^{\mathrm{d}}$ Fisher's exact test

\begin{tabular}{lcccccc}
\hline & \multicolumn{2}{l}{ Early AMD eyes $(n=48)$} & & $P$-value \\
& \cline { 2 - 4 } & All & CD $(n=28)$ & SDD $(n=20)$ & & \\
$(n=42)$ & & \\
\hline $\begin{array}{l}\text { 1-mm GCL-IPL } \\
\text { thickness }(\mu \mathrm{m})\end{array}$ & $19.6 \pm 7.1$ & $20.1 \pm 7.4$ & $18.9 \pm 6.7$ & 0.6 & $17.3 \pm 3.2$ & 0.1 \\
$\begin{array}{l}\text { 3-mm GCL-IPL } \\
\text { thickness }(\mu \mathrm{m})\end{array}$ & $40.0 \pm 5.5$ & $41.4 \pm 5.0$ & $37.9 \pm 5.6$ & 0.03 & $45.1 \pm 3.4$ & 0.04 \\
$\begin{array}{l}\text { 6-mm GCL-IPL } \\
\text { thickness }(\mu \mathrm{m})\end{array}$ & $28.4 \pm 2.9$ & $28.9 \pm 2.7$ & $27.6 \pm 3.1$ & 0.2 & $31.2 \pm 2.3$ & 0.03 \\
$\begin{array}{l}\text { 1-mm CT }(\mu \mathrm{m}) \\
\text { 3-mm CT }(\mu \mathrm{m})\end{array}$ & $212.4 \pm 78.4$ & $214.3 \pm 69.2$ & $209.6 \pm 91.6$ & 0.9 & $280.6 \pm 71.9$ & 0.001 \\
6-mm CT $(\mu \mathrm{m})$ & $193.0 \pm 61.8$ & $196.4 \pm 58.8$ & $188.4 \pm 67.1$ & 0.7 & $234.9 \pm 60.9$ & 0.04 \\
\hline
\end{tabular}

${ }^{a}$ Data analysis results comparing eyes with CD vs. eyes with SDD. Measurements between groups were compared using the general linear model, including age, sex, axial length, and spherical equivalents as covariates

${ }^{\mathrm{b}}$ Data analysis results comparing eyes with age-related macular degeneration (AMD) vs. control eyes. Measurements between groups were compared using the general linear model, including age, sex, axial length, and spherical equivalents as covariates 

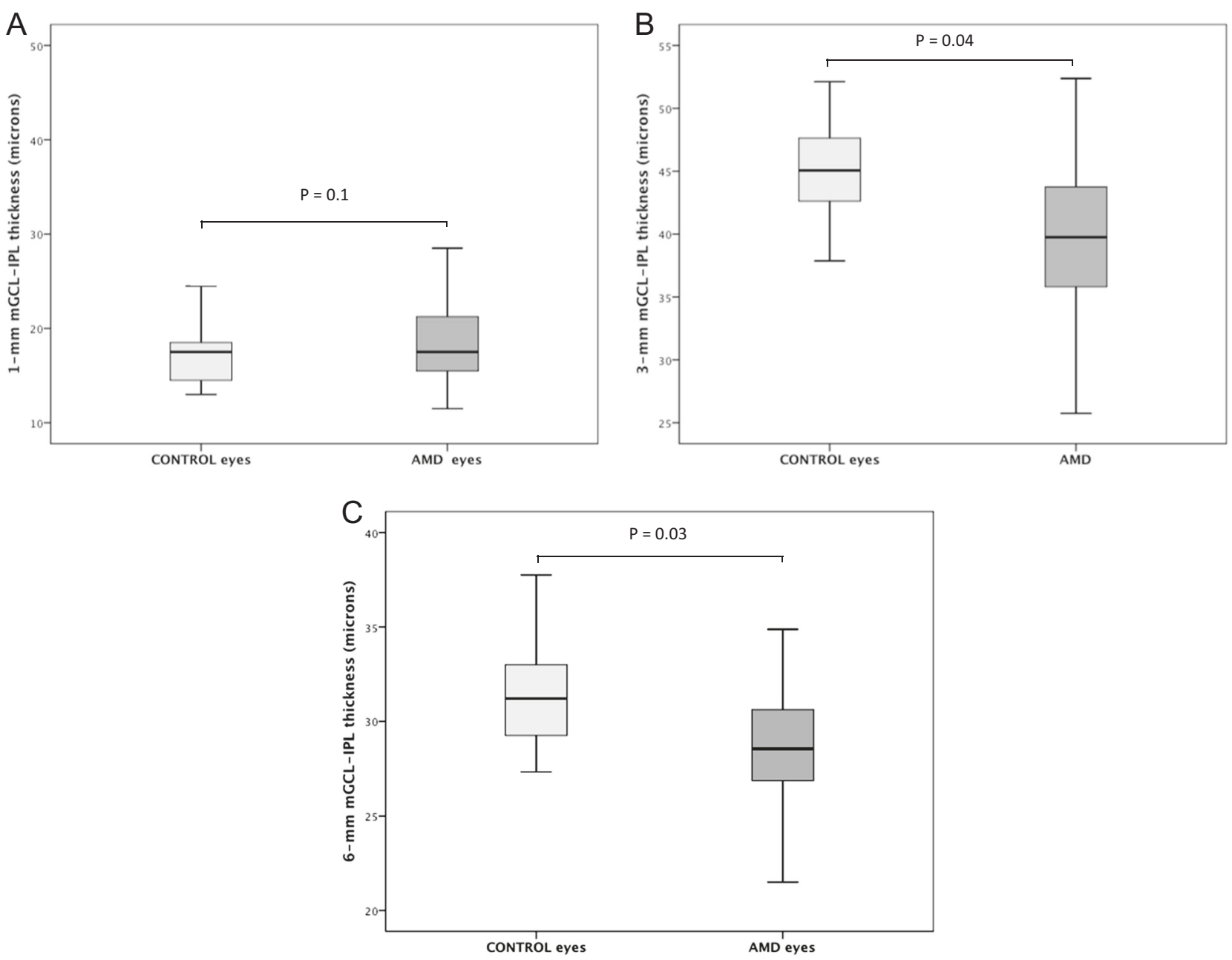

Fig. 2 Box-plots of macular ganglion cell layer-inner plexiform layer (mGCL-IPL) thickness in eyes with early age-related macular degeneration and healthy controls in the 1,3, and $6 \mathrm{~mm}$ ETDRS areas; $(\mathbf{a}, \mathbf{b}$, and $\mathbf{c})$, respectively. $P$-values shown in the figure result from the general linear model analysis adjusted for sex, age, axial length, and spherical equivalent as covariates

There was a significantly decreased GCL-IPL thickness at 3-mm and 6-mm in patients with early AMD compared to controls, with a reduction of 11.5 and $9 \%, 45.1 \pm 3.4$ vs. $40.0 \pm 5.5$ and $31.2 \pm 2.3$ vs. $28.4 \pm 2.9$ for the $3-\mathrm{mm}$ and 6 mm-area, respectively, $P<0.05$ (adjusted for age, sex, axial length, and spherical equivalent). The GCL-IPL thickness at $3 \mathrm{~mm}$ was significantly reduced in eyes with SDD with respect to eyes with drusen alone $(41.4 \pm 5.0$ vs. $37.9 \pm 5.6$; $P=0.03$, adjusted for sex, age, axial length, and spherical equivalent), with a reduction of $8.5 \%$. (Figs. 2 and 3 )

There was no correlation of any thickness values with best-corrected visual acuity.

\section{Discussion}

The present study showed that choroidal thickness, and the macular ganglion cell layer-inner plexiform layer thickness in the 3 and $6 \mathrm{~mm}$ areas is reduced in early AMD with respect to normal eyes. The GCL-IPL layer is thinner in eyes with SDD with respect to eyes with drusen alone.

The pathogenesis of SDD is still not completely understood. A recent review by Sivaprased et al. discussed parainflammatory factors and heightened immune response mechanisms, which lead to a reduced ability of the retina to regulate damage from external noxious insults or endogenous tissue and cell stress [6]. Other hypotheses are based on mitochondrial decline and retinal pigment epithelium/ photoreceptor damage linked to oxidative stress and lipid peroxidation with aging [6]. Recent investigations have shown a stronger association of SDD with retinal angiomatous proliferation, and geographic atrophy, and a reduction of choroidal thickness $[5,6,11,12]$.

Many authors have reported thinning of the choroid in eyes with SDD, as observed with OCT, but it is not yet clear if choroidal vasculature alterations are a primary pathogenetic factor $[5,6,11,12,16,20,21]$. Our data did not show significant choroidal thinning in eyes with SDD. This could 

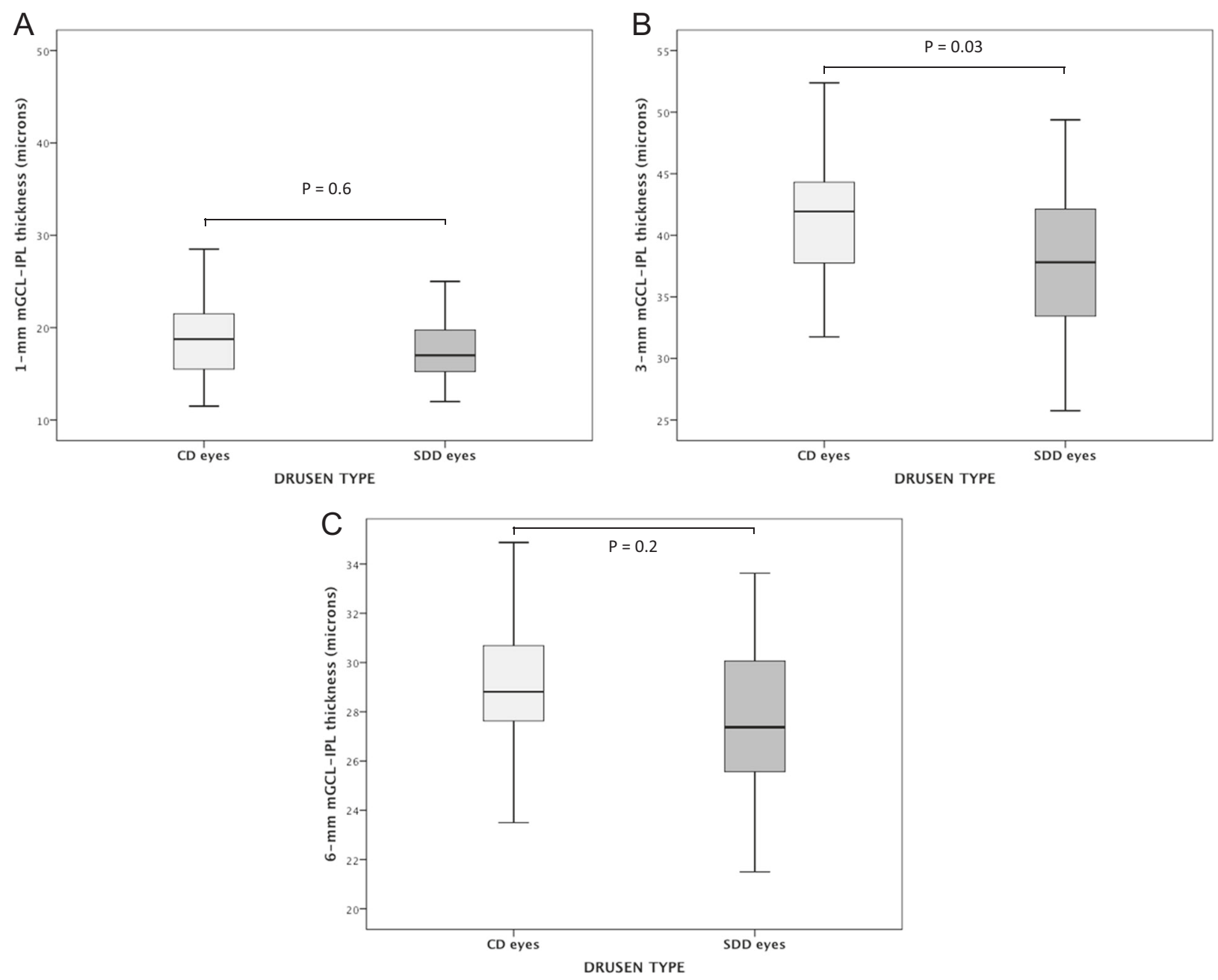

Fig. 3 Box-plots of macular ganglion cell layer-inner plexiform layer (GCL-IPL) thickness in eyes with conventional drusen (CD) and subretinal drusenoid deposits (SDD), $(\mathbf{a}, \mathbf{b}$, and $\mathbf{c}$ ), respectively. $P$-values shown in the figure result from the general linear model analysis adjusted for sex, age, axial length, and spherical equivalent as covariates

be due to differences in SDD classification in our study with respect to other reports and also owing to the association of drusen with SDD in most of the eyes in our cohort. Indeed, SDD are frequently found in association with drusen and Lains et al., similar to our results, reported on 186 eyes with AMD where $47.3 \%$ of eyes had drusen alone, $5.4 \%$ had SDD alone, and $45.2 \%$ had both SDD and drusen [9]. In a recent study on 651 subjects, a major finding was that SDD were frequently present in patients with early AMD in 49\% of cases and in intermediate AMD in 79\% of patients [8]. Furthermore, Sarks et al. reported that only $2.7 \%$ of their cases with SDD did not have signs of AMD [10].

Vongkulsiri et al. studied the relationship between SDD and large choroidal vessels but did not find any correlations [20]. Spaide et al. used enface SDOCT but did not report diffuse alterations of the choriocapillaris layer [12]. However, recently it was reported that choroidal filling defects during angiography were more frequent in eyes with pseudodrusen [21]. Mrejan and Spaide, retrospectively investigated highly myopic eyes with reduced choroidal thickness but did not find development of SDD concluding that there is no conclusive evidence to support a causative relationship between choroidal thickness and SDD formation [16].

Our results of GCL-IPL thinning in early AMD are in agreement with those of other authors [3, 22, 23]. The choroid and the GCL-IPL become thinner with age and are positively correlated [13]. The oxygen supply to the retinal layers depends on the retinal and choroidal circulation [24]. It has been hypothesized that hypoperfusion and chronic vasculature failure due to macular aging are determining factors in post receptor damage [1, 25-27]. In advanced AMD, photoreceptor damage could chronically reduce input to the inner retinal layers and cause trans-neural degeneration leading to apoptosis of neurons [3]. Studies in the animal model have shown impairment of the inner plexiform and ganglion cell layer in retinal degeneration [27]. Zucchiatti et al. reported GCL-IPL thinning in patients 
with early intermediate AMD [3]. Similar to our findings of thinning of the GCL-IPL in the 3 and $6 \mathrm{~mm}$ ETDRS areas, Lee et al. reported an annular pattern of GCL-IPL thinning around the fovea in early AMD [23]. This annular pattern could be linked to the anatomical structure of the macula, which shows gradual thinning of the inner retinal layers, including the vascularized retinal layers, as they approach the fovea to terminate in the central foveal avascular zone [27]. The annular pattern could also be linked to the vascular supply characteristics of the foveal vs. the perifoveal region. The superficial retinal vascular network predominantly supplies the ganglion cell layer whereas the deep vascular plexus, prevalently of the inner nuclear layer, constitutes the perifoveal vascular ring and terminates closer to the central fovea [28].

It is difficult to speculate on the reasons for a thinner perifoveal GCL-IPL in patients with SDD as this is a novel finding and requires further investigation. Lee et al. found an inverse relationship between drusen load and average GCL-IPL thickness [23] but, to our knowledge, there is no standardised methodology to calculate SDD load. The preferential site of localization of SDD in the rod rich perifoveal areas may be implicated $[5,7,8]$ and our results showed that the GCL-IPL thickness in the perifoveal area is significantly reduced. Interestingly, SDD are found at sites of choroidal watershed zones, suggesting a possible topographical hypoperfusion pathogenesis $[29,30]$. If choroidal alterations and thinning do play a causative role in SDD formation, we could tentatively speculate that thinning of the GCL-IPL layer, which is correlated with choroidal thickness [13], could be part of a generalised spectrum of vascular alterations of the choroid and retina. SDD could be a manifestation of enhanced hypoperfusion and chronic vasculature failure of macular aging associated with AMD [1, 25-27]. This conceptive hypothesis could be worthy of reflection and could be supported by the fact that SDD is encountered in older individuals and that neovascularisation and geographic atrophy are more frequently associated with the condition.

Studies on a larger number of patients using OCT angiography are warranted in order to evaluate the deep and superficial vascular networks in SDD. Most importantly, longitudinal investigation is necessary to evaluate the implications of choroidal thinning and thinning of the vascularised retinal layers in the pathogenesis of SDD.

We acknowledge that our study has some shortcomings in that we enrolled a limited number of patients. In order to measure choroidal thickness, we used a semi automatic method, which however, had previously shown good interobserver repeatability. Finally, our study cannot clarify the cause-effect relationship of choroidal and retinal layer thinning in the pathogenesis of SDD as this would require further research and longitudinal analysis.

\section{Summary}

\section{What was known before}

- Recent classification of early age-related macular degeneration differentiates lipoprotein extracellular accumulations in drusen localized below the retinal pigment epithelium from SDD localized above the retinal pigment epithelium.

- The pathogenesis of SDD is not completely understood although choroidal thinning is reported.

\section{What this study adds}

- In eyes with early age-related macular degeneration associated with SDD there is thinning of the macular ganglion cell layer-inner plexiform layer.

- This layer is vascularised and correlates positively with choroidal thickness suggesting a possible pathogenetic implication of generalised choroid/retinal vascular alterations.

\section{Compliance with ethical standards}

Conflict of interest The authors declare that they have no conflict of interest.

Publisher's note: Springer Nature remains neutral with regard to jurisdictional claims in published maps and institutional affiliations.

\section{References}

1. Feigl B, Brown B, Lovie-Kitchin J, Swann P. Functional loss in early age-related maculopathy: the ischaemia post receptoral hypothesis. Eye (Lond). 2007;21:689-96.

2. Shin IH, Lee WH, Lee JJ, Yo YJ, Kim JY. Thickness of the macula, retinal nerve fiber layer and ganglion cell-inner plexiform layer in the age-related macular degeneration. Retina. 2018;38:252-62.

3. Zucchiatti I, Battaglia Parodi MB, Pierro L, Cicinelli V, Gagliardi $\mathrm{M}$, Castellino N, et al. Macular ganglion cell complex and retinal nerve fiber layer comparison in different stages of age-related macular degeneration. Am J Ophthalmol. 2015;160:602-7.

4. Spaide RF. Improving the age-related macular degeneration construct. Retina. 2018;38:891-9.

5. Spaide RF, Ooto S, Curcio CA.Subretinal drusenodi deposits AKA pseudodrusen.Surv Ophthlmo. 2018;63:728-815.

6. Sivaprasad S, Bird A, Nitiahpapand R, Nicholson L, Hykin P, Chatrizalli I. Perspectives on reticular pseudodrusen in age-related macular degeneration. Surv Ophthalmol. 2016; 16:521-37.

7. Curcio CA, Messinger JD, Sloan KR, McGwin G, Medeiros NE, Spaide RF. Subretinal drusenoid deposits in non-neovascular agerelated macular degeneration: morphology, prevalence, topography, and biogenesis model. Retina. 2013;33:265-76. 
8. Zarubina AV, Neely DC, Clark ME, Huisingh CE, Samuels BC, Zhang Y, et al. Prevalence of subretinal drusenoid deposits in older persons with and without age-related macular degeneration, by multimodal imaging. Ophthalmology. 2016;123:1090-1100.

9. Lains I, Wang J, Providencia J, Mach S, Gil P, Gil J, et al. Choroidal changes associated with subretinal drusenoid deposits in age-related macular degeneration using swept source optical coherence tomography. Am J Ophthalmol. 2017;180:55-63.

10. Sarks JP, Sarks SH, Killingsworth MC. Evolution of geographic atrophy of the retinal pigment epithelium. Eye (Lond). 1988;2:552-77.

11. Switzer DW Jr, Mendonca LA, Saito M, Zweifel SA, Spaide RF. Segregation of ophthalmoscopic characteristics according to choroidal thickness in patients with early age-related macular degeneration. Retina. 2012;32:1265-71.

12. Spaide RF. Colocalization of pseudodrusen and subretinal drusenoid depostis using high-density en face spectral domain optical coherence tomography. Retina. 2014;34:2336-45.

13. Abdolrahimzadeh S, Parisi F, Scavella V, Recupero SM. Optical coherence tomography evidence on the correlation of choroidal thickness and age with vascularized retinal layers in normal eyes. Retina. 2016;36:2329-38.

14. Zweifel SA, Immamura Y, Spaide TC, Fujiwara T, Spaide RF. Prevalence and significance of subretinal drusenoid deposits (reticular pseudo-drusen) in age-related macular degeneration. Ophthalmology. 2010;117:1775-81.

15. Spaide RF, Curcio CA. Characterization with multimodal imaging. Retina. 2010;30:1441-54.

16. Mrejen S, Spaide RF. The relationship between pseudodrusen and choroidal thickness. Retina. 2014;34:1560-6.

17. Abdolrahimzadeh S, Parisi F, Plateroti AM, Evangelista F, Fenicia V, Scuderi G, et al. Visual acuity, and macular and peripapillary thickness in high myopia. Curr Eye Res. 2017;42:1468-73.

18. Abdolrahimzadeh S, Felli L, Plateroti AM, Perdicchi A, Contestabile MT, Recupero SM. Spectral domain optical coherence tomography evidence of retinal nerve fibre layer and ganglion cell loss in adult patients with neurofibromatosis type 1. Retina. 2016;36:75-81.

19. Staurenghi G, Sadda S, Chakravarthy U, Spaide RF. Proposed lexicon for anatomic landmarks in normal posterior segment spectral-domain optical coherence tomography. Ophthalmology. 2014;121:1572-8.

20. Vongkulsiri S, Oto S, Mrejan S, Suzuki M, Spaide RF. The lack of concordance between subretinal drusenoid deposits and large choroidal blood vessels. Am J Ophthalmol. 2014;158:710-5.

21. Zhou Q, Daniel E, Grunwald JE, Maguire MG, Gewally DY, Martin DF, et al. Association between pseudodrusen and delayed patchy choroidal filling in the comparison of age-related macular degeneration treatments trials. Acta Ophthalmol. 2017;95:e51820.

22. Savastano MC, Minnella AM, Tamburino A, Govinco G, Ventre S, Falsini B. Differential vulnerability of retinal layers to early age-related macular degeneration: evidence by SD-OCT segmentation analysis. Invest Ophthalmol Vis Sci. 2014;55:560-6.

23. Lee EK, Yu HG. Ganglion cell-inner plexiform layer and peripapillary retinal nerve fiber layer thicknesses in early age-related macular degeneration. Invest Ophthalmol Vis Sci. 2015;56:397683.

24. Arden G, Sidman R, Arap W, Schlingemann R. Spare the rod and spoil the eye. Br J Ophthalmol. 2005;89:764-9.

25. Yu DY, Cringle S. Oxygen distribution and consumption within the retina in vascularised and avascular retinas and in animal models of retinal disease. Prog Ret Eye Res. 2001;20:175-208.

26. Cringle S, Yu DY, Tu P, Su EN. Intraretinal oxygen consumption in the rat in vivo. Invest Ophthalmol Vis Sci. 2002;43:1922-7.

27. Spaide RF, Curcio CA. Evaluation of segmentation of the superficial and deep vascular layers of the retina by optical coherence tomography angiography instruments in normal eyes. JAMA Ophthalmol. 2017;135:259-62.

28. Snodderly DM, Welnhaus RS, Choi JC. Neural-vascular relationships in central retina of macaque monkeys (Macaca fascicularis). J Neurosci. 1992;12:1169-93.

29. Grewal DS, Chou J, Rollins Sd, Fawzi AA. A pilot quantitative study of topographic correlation between reticular pseudodrusen and the choroidal vasculature using enface optical coherence tomography. PLoS ONE. 2014;9:e92841.

30. Alten F, Heiduschka P, Clemens CR, et al. Localised reticular pseudodrusen and their topographical relation to choroidal watershed zones and changes in choroidal volumes. Invest Ophthalmol Vis Sci. 2013;54:3250-7. 